\title{
LIMITING FACTORS IN WIDESPREAD Adoption of ACTIVE QUEUE MANAGEMENT IN THE PHILIPPINES' CONSUMER ELECTRONICS SPACE
}

\begin{abstract}
Min Guk I. Chi
Bachelor of Business Administration, S P Jain School of Global Management

ABSTRACT

The premise that Active Queue Management (AQM) is effective in both quantitative and qualitative settings in residential and enterprise networks has repeatedly been established in multiple papers from academic journals along with private studies in addressing bufferbloat, characterized as excessive latency because of heavy network utilization. However, the presence and understanding of bufferbloat mitigation is absent and not well-known in the Philippine Internet of Things space except enthusiasts, willing to take the time to examine the concept along with its benefits. Hence, this paper examines possible reasons as to why AQM is not widely adopted by Philippine consumers and industries in increasing productivity considering the COVID-19 Pandemic: a lack of basic understanding of bufferbloat and its implications, the complexity of the concept, the know-how required to execute its implementation being far too high, and the lack of perceived benefit by existing telecommunications players in the country.
\end{abstract}

\section{KEYWORDS}

Active Queue Management, Consumer Adoption, COVID-19, Bufferbloat.

\section{INTRODUCTION}

In an increasingly digital world, a strong and robust internet infrastructure is paramount; this is more so considering the context in which this paper was made: during the Severe Acute Respiratory Syndrome - Coronavirus 2 pandemic, colloquially known as COVID-19. With major events around the world being moved to a virtual medium considering the virus spreading through respiratory droplets, the internet is increasingly utilized to compensate for productivity in many fields, including but not limited to the academe and commercial - events that generally can be held from the comfort of an individual's home. Hence, the need for a robust internet is essential since any further disruptions will increase the losses of productivity that have been incurred due to the global pandemic.

This premise is given weight thanks to the medium of these events: video conferencing applications such as Zoom have risen to prominence thanks to the need for virtually distant conferences. Considering this, video conferencing is a latency-sensitive application which requires that the latency of the internet is kept at a minimum to avoid video and audio degradation. Additionally, latency-sensitive activities such as Voice over IP (VoIP), Video Streaming, and Low Latency Online Gaming are some of the other examples where sudden increases in latency prove significantly detrimental. This phenomenon in internet networks is 
known as bufferbloat; according to DSLReports, this is characterized as "the undesirable latency caused by routers and cable/DSL modems buffering more data than necessary." [5]

One of the mitigations that is present thanks to the Institute of Electrical and Electronics Engineers (IEEE) is Active Queue Management (AQM), characterized as the management of data packets via proactively dropping packets before it exceeds the buffer, preventing excessive latency thanks to heavy load. Therefore, this study seeks to examine the reasons as to why AQM is noticeably absent in the Internet of Things: consumer electronics space despite the clear benefits of its applications in existing network infrastructure.

With that said, the primary contributions of this paper are as stated:

i. First, it provides an analysis of consumer preferences in the Philippines in terms of price, purchase behavior, and factors that may consider a purchase of a third-party router in order to solve their problem of bufferbloat.

ii. Second, it shows the current level of understanding of Filipinos on what bufferbloat is and what are the effective mitigations in solving such a problem, or lack thereof.

iii. Third, it provides possible opportunities into hastening the adoption of Active Queue Management strictly in terms of a business perspective along with causing disruption in the Philippine space.

a. By extension, it provides possible product offerings of third-party routers at reasonable price points in the context of the Philippines.

iv. Lastly, the paper is an attempt into bridging the concept of Active Queue Management into the applied space. More specifically, Active Queue Management being given in the hands of consumers and allowing for a better internet experience overall, thus consumers staying relevant in the Fourth Industrial Revolution.

\section{REVIEW OF RELATED WORK}

\subsection{Robust Telecommunications Industry}

According to Chi (2020) [1], the paper asserts that modern telecommunications are essential in today's technologically adept population, along with the premise that higher internet speeds are positively correlated with the economic status of a country. With these two statements, this gives a clear foundation for the basis of the introduction - a robust infrastructure being a must. Additionally, on the same paper, it asserts that the improvement of telecommunications is a must to provide better opportunities for all Filipinos who increasingly rely on access to the internet in order to have a chance at improving their quality of life; with this, further pretext is given to the necessity of a strong base for the telecommunications industry. Without a strong industry, the mitigations given to solve bufferbloat will have minimal impact if the internet constantly goes out thanks to poor reliability from internet providers. Furthermore, the impact of these antibufferbloat mitigations is also lost if the power grid infrastructure is not fully developed. Power outages will cause a loss of internet given the logical premise that these are connected to electricity. Hence, in conclusion, this source proves relevant given the foundation it provides for the requirements needed to give AQM a consistently meaningful impact.

\subsection{Active Queue Management}

The concept of Active Queue Management in and of itself must be well-defined given that said concept is the core foundation on which this research lies. For instance, the concept was formalized into actual use by the Institute of Electronics and Electrical Engineers in 1993 
according to Adams (2013)[12]; from this, conclusions can be inferred. One, with respect to the context in which AQM was appearing in research papers, it can be opined that signs for the internet were already on the point for civilian use. Coincidentally, this was also the time where Berners-Lee introduced the concept of the World Wide Web (WWW): a way for the internet to be bridged from military use to general civilian use. Hence, with the increase in data packets moving because of the increased reach of civilians using the internet, the problem of buffers being full quickly due to said increase in data packets leading to internet degradation would arise. Therefore, because of this, AQM models have been examined by multiple organizations seeking to solve the problem.

Initial frameworks that involved the AQM system during the 1990s-2000s were the Tail Drop and the Random Early Detection algorithms. These are characterized as two methods in which packets that are incoming whilst the buffer is full is effectively dropped. This means that the data incoming will not be accepted into the current buffer until the buffer can allocate can process the existing data packets. The tail-drop method has its limitations: for instance, this method is a passive method of AQM. Based on a previous lecture on internet protocols from NC State University in 2014, "Tail drop is a passive queue management algorithm. In this algorithm, the traffic is not differentiated, and each packet is at the same priority. Also, the main consideration here is the maximum queue length at each router and its services using the first in first out algorithm." This effectively does not differentiate between latency-sensitive packets and only adjusts the buffer once it's full. Hence, it has a significant shortcoming since it does not factor in differing types of packets that require a higher priority than others [9]. According to a paper published by the IEEE in 2002 [3], Random Early detection is inherently limited considering that RED is inflexible with respect to the paper mentioning "A drawback in deploying RED stems from its apparent tuning difficulties. As we now show, we believe this difficulty stems in large part to RED's use of average queue length." Since it uses an average, limitations inherent to using an average are applicable such as susceptibility to extremes and inaccuracies in data throughput to make the RED algorithm tailor-made to the concerned network.

More developed frameworks have been developed to compensate for the weaknesses of the initial algorithms as mentioned above. For instance, the development of Fair Queue - Controlled Delay (FQ - CoDel) by Jacobson and Nichols in 2012 non-exhaustively improves on the initial frameworks of Tail-Drop and RED on the following [10]:

1. It is parameter-deficient, meaning that it is inherently easier to configure considering networks with dynamic throughput.

2. CoDel also can determine certain types of traffic: those that cause bufferbloat, and those that don't. a. Those that don't are effectively ignored by the AQM algorithm, whilst those that are will be subjected to the algorithm to minimize delay as much as possible.

3. Implementation is simple, so it can be utilized in consumer-grade products and in highend networking hardware.

Given the effectiveness of FQ - CoDel in reducing bufferbloat, a study has been made to examine the feasibility of implementation into network equipment for commercial use. A study conducted by White and Rice in 2013 [16] has shown significant benefits when correctly implementing the use of CoDEL and other AQM implementations such as Proportional-Integral Controller, that was mentioned above, and Stochastic Fair Queuing with CoDel. Surprisingly, AQM is already present in existing cable modems as of the time of this research paper and was just not implemented by operators; when configured optimally, it has been quantitatively and qualitatively shown that improvements in latency are significant. This, however, is only limited to upload speeds and does not examine the download side, what many households use. Hence, 
this paper is valuable in proving that the theoretical algorithms do make a meaningful difference in internet experience.

Over the years, research has been made to see which AQM algorithm is the most optimized for network traffic. With the current AQM implementations Stochastic Fair Queuing was the best algorithm for implementation of reducing excessive delay when it comes to heavy network utilization [7]. This is relevant considering that there have been many AQM implementations that have been proposed by researchers across the world, seeking to solve the issue of bufferbloat.

However, while attempting to find the best AQM algorithm available, there has been a clear lack of standardization of metrics to quantitatively measure the results of researchers' algorithms to determine what algorithm is best all-around in order to see which implementation of AQM should be actively used in the real-world. The general metrics that developers of future AQM algorithms should use to benchmark performance. Specifically, they recommend merging the processes of Analytic Hierarchy Process (AHP) along with Technique in Order of Preference by Similarity to the Ideal Solution (TOPSIS) to create a new benchmarking standard that is ideal for new AQM algorithms to be tested against: promoting robustness and quality (Khatari et.al., 2019) [7].

Perhaps another clear application of the effectiveness of AQM is in big data: significant amounts of data running through a server at once. Given that big data requires quick execution of data processing with mass inputs into meaningful outputs to be of use to experts, it also requires that the connection to the internet for this is responsive (i.e., latency-free). Hence, an examination was made to see whether AQM would provide a tangible benefit in Hadoop clusters and in the MapReduce Programming Model. Given the analysis of the research that has been done, it has also shown that bufferbloat can be reduced significantly, by $85 \%$ whilst only increasing the Hadoop execution time using the MapReduce Model by 5\%. However, it strongly cautions that getting the configuration right is the only way to achieve optimal results, as poor configurations lead to increases in Hadoop executions and non-ideal reductions in bufferbloat. Hence, it can be concluded that AQM is clearly scalable in many settings, from the confines of one's home to the large data processing units that are used by technology companies to process large amounts of information. [13]

As of today, in open-source software, AQM has more developments. In OpenWrt, an open-source Linux base for networking hardware such as routers and wireless extenders, the implementation of Cake (Common Applications Kept Enhanced) — from the Bufferbloat community [2] — has provided for further improvements in solving this problem. This discipline for queuing network packets considers AQM as only one of the measures necessary to address bufferbloat. Cake includes the following:

1) Traffic Shaper

2) Priority Queue

3) Flow Isolation

4) AQM

5) Packet Management

All these factors lead to reduced latency in general internet use, especially in latency-sensitive applications and has factored in additional information that makes the difference between a lowlatency internet and one that is significantly crippled by bufferbloat. Additionally, cake is superior to CoDel, non-exhaustively, in a couple of ways: 
1) Command Line interface is simpler than CoDel.

2) Reduced CPU Load thanks to an integral shaper.

3) Explicit Congestion Notification (ECN) is always on, avoiding false positives.

4) Ease of availability; a. Since it is on Linux, an open-source platform, it is easily available with commands understood on the platform.

With all of this, it shows the progression of AQM into actual practice and is relevant to gain a better understanding of the merits of applying this into the consumer market, especially considering the COVID-19 pandemic, and its impacts still forcing many events and industries to go virtual, as any sign of latency means missed productivity which often proves to be major.

\subsection{Consumer Behavior}

For instance, Consumer Behavior: 11th Edition [14] has relevant concepts that are of value. When it comes to effectively appealing to consumers, there are four elements to properly understand consumer behavior: Motives, Cues, Responses, and Reinforcement. These are characterized as:

1. Motives: The incentive behind doing something.

2. Cues: The mechanism in which consumers will know that a certain product/service is what they need (e.g., Marketing, Advertising).

3. Responses: How the consumer reacts to the former two factors.

4. Reinforcement: The way in which consumers are solidified towards believing a certain view.

In terms of how to create effective advertising, there are many effective avenues: comparative advertising, appeal to humor, fear, and sexual appeal. These avenues are defined as follows:

1. Comparative Advertising: By making claims that one's product is superior to the competition, this appeals to consumers since they only want what's best for them.

2. Appeal to Humor: Using advertising to induce laughter, it promotes a positive brand image and leaves a long-term impact.

3. Appeal to Fear: By presenting a clear threat that consumers should be fearful of, and providing a solution to said fears, it promotes consumers to buy your products for a sense of safety.

4. Sexual Appeal: Through this method, consumers are captivated by an ideal human image. This, however, requires careful execution. Else, consumers will only be captivated on the model and not the product.

Extending on consumer behavior, another research paper proves its value given the recency of the paper. A paper made by Moon, Choe, and Song in 2021 [8] describes consumer behavior in South Korea considering the COVID-19 pandemic; most respondents would prefer to acquire their goods online rather than getting goods in person considering personal safety. With this, it can be argued via the use of the Protection-Motivation Theory; this is simply explained as protection being the first reason for their actions and with that framework in mind, people will gravitate their decisions for the sake of protection.

Another relevant part of a business is pricing and how deciding how much or little a product or service is priced has a meaningful impact on whether a consumer would purchase said product or service. Given a research paper by Quan, Quan, and Wang in 2019 [11], consumers' expectations play a factor as to influencing pricing. On one hand, if consumers' expectations are very high for the product in question and prioritize psychological satisfaction, the price is effectively damaged 
and the same goes with profits. This is because a higher standard is required - and this compels the seller to reduce prices to not disappoint them. On the other hand, if a consumer is only expecting the bare minimum and does not prioritize their happiness, prices can rise and profits by extension. This is due to their perspective on only focusing with the product in question getting the job done. Given that one of the goals of this paper is to create a potentially disruptive business, the goal is to reach as many customers as possible.

Lastly, of relevance is consideration for how consumers are influenced in terms of their purchases specifically with Internet of Things devices. There are certain factors that play a larger role in a consumer as to whether they will buy a product under this category i.e., most important is trust. When a consumer has confidence in a brand's products and services through superior customer experience, perceived ease of use, and a proven track record, adoption is significantly hastened. On the other, factors such as social influence do play a part though to a lesser extent against the factors (Tsourela \& Nerantzaki, 2020). [15]

\section{RESEARCH FRAMEWORK}

With the available literature, the concept of Active Queue Management itself is only available with no research papers on examining the actual limitations of full market adoption; hence, this warrants the use of an individual framework unique to this research to fully ascertain the reasons for the lack of market adoption. Given that the market is the body that is directly concerned with the apparent lack of adoption, marketing fundamentals make the most sense to apply as a theoretical framework.

Regarding the use of marketing fundamentals, parts of a market plan - according to Pearson's 17th Edition of Principles of Marketing [6] - make most sense in applying here; for instance, parts of this paper can already be used for the current marketing situation such as the market description, existing products, and competition. Marketing actions will be manifested as recommendations. Essentially speaking, this is a market reach problem, basics in marketing are used for most of the paper to solve the question of the lack of market adoption on the internet of things - consumer electronics space.

In this case, the foundational framework that is most applicable will be thematic analysis. Considering the nature of the research question which requires that factors be fleshed out to sufficiently answer the question, dividing the independent variables (factors) into themes will be of use to the fulfillment of this research paper. Hence, there will be no use of hypothesis testing when it comes to the information that has been obtained along with corresponding analysis and discussion as a result.

Consequently, given no hypothesis testing, what the data will be used for is to determine the extent of the veracity of the hypotheses that will be mentioned in the latter part of this paper. In other words, the goal is to determine to what extent the hypotheses are true in the context of the data that has been obtained, and whether said data confirms or rejects the possible factors that answer the research question at hand.

\section{Methodology}

The primary mechanism to successfully answer the research question will primarily be done through data collection; through the input of respondents, information relevant to the research objectives will be revealed via a survey questionnaire. A secondary method will be via the use of existing literature from relevant and quality sources. 


\section{Respondent Profile \& Sample}

Information obtained from respondents such as age, employment, income bracket, will provide relevant information as to how they are influenced in their purchase decisions and their preferences in the context of this study.

For the purposes of this study, a sample of 200 respondents will be obtained from multiple areas of the country (in Luzon, Visayas, and Mindanao) to provide a diverse picture of their perceived wants to promote an ideal internet experience.

\section{Data Collection Method}

The variables in this paper will be ascertained via an online survey which is distributed to respondents. A prototype of the survey has already been made to ensure that the base version is fully functional; hence, a final version was made with questions that have been tweaked to be more relevant to the study. Consequently, the sampling technique that was used for this paper is non-probability based; in particular, convenience and snowball sampling are the prevalent mechanisms used.

\section{Questionnaire\& Tools}

Survey questions are segmented into five parts which contain questions that ask for certain information; the Likert scale was often used to determine the respondents' inclinations to agreeing or disagreeing to certain statements. Additionally, open-ended questions were used to factor in a variety of responses that respondents may give to certain questions which requires to be flexible about the context of the respondent.

Data that has been obtained through the questionnaire will be analyzed via IBM's SPSS and Microsoft Excel with PH Stat 4.1 in order to tabulate, organize, and analyze the data; the primary mechanism of analysis is through the cluster method: done in order to create a customer profile; three segments have been made for the sake of the objectives mentioned above. Standard statistical treatment will also be utilized: Sample Mean, Median, Mode, Standard Deviation; this will be relevant in analysis of the data that has been given by respondents and will promote a representative picture of the market to see whether or not a business undertaking is feasible. For the purposes of keeping the paper concise, only the customer profiles from the cross-tabulation will be shown. All other data will be available for viewing in the original paper.

\section{RESUlts}

\begin{tabular}{|c|c|c|}
\hline Segments & Characteristics \&Needs & Solution \\
\hline $\begin{array}{l}\text { Budget } \\
\text { Consumer }\end{array}$ & $\begin{array}{l}\text { a. A Monthly Income of } \\
\text { Minimum Wage Up } \\
\text { Until Less Than } \mathrm{P} 22,000 . \\
\text { b. Spending } \text { P500 - 尹2,000 } \\
\text { for Internet Usage } \\
\text { Monthly. } \\
\text { c. Knows Little to } \\
\text { Moderate Knowledge on } \\
\text { Bufferbloat, And. } \\
\text { d. Prefers Buying } \\
\text { Technology Products }\end{array}$ & $\begin{array}{l}\text { A Budget Router with a Low Production } \\
\text { Cost and Low Sale Cost is Attractive. } \\
\text { Must be Cheap but Robust in Terms of } \\
\text { Build Quality. } \\
\text { Low Specifications: } \\
\text { Basic Single-Core, Low Frequency CPU } \\
8 \text { MB of Flash } \\
64 \text { MB of RAM }\end{array}$ \\
\hline
\end{tabular}




\begin{tabular}{|c|c|c|}
\hline & Physically. & $\begin{array}{l}\text { Providing Basic Wi-Fi Standard } \\
\text { (802.11ac); } \\
\text { Wireless Protected Access } 2 \text { (WPA 2) } \\
\text { Low-Powered Antennas: } \\
\text { Maximum of } 20 \text { decibel-milliwatts (dBm) }\end{array}$ \\
\hline $\begin{array}{c}\text { Mid- } \\
\text { Range } \\
\text { Consumer }\end{array}$ & $\begin{array}{ll}\text { a. A Monthly Income of } \\
\text { 尹22,000 Up Until Less } \\
\text { Than } \text { 尹132,000. } \\
\text { b. Spending P2,000 - } \\
\text { P5,000+ for Internet } \\
\text { Usage Monthly. } \\
\text { c. Knows Little to } \\
\text { Moderate Knowledge on } \\
\text { Bufferbloat, And. } \\
\text { d. Prefers Buying } \\
\text { Technology Products } \\
\text { Physically. }\end{array}$ & $\begin{array}{l}\text { A Mid-Range Router with Slightly Higher } \\
\text { Production Cost and Increased Price is } \\
\text { Attractive: } \\
\text { An Increased Budget for Specifications } \\
\text { and Better Build Design. } \\
\text { Medium Specifications: } \\
\text { Single Core, Higher Frequency CPU } \\
\text { 8-16 MB Flash } \\
\text { 64-128 MB RAM } \\
\text { Providing Slightly Better Wi-Fi Standard } \\
\text { (802.11ac): } \\
\text { Wireless Protected Access } 2 \text { (WPA 2) } \\
\text { Medium Powered Antennas } \\
\text { Maximum of } 25 \text { decibel-milliwatts (dBm) }\end{array}$ \\
\hline $\begin{array}{l}\text { High-End } \\
\text { Consumer }\end{array}$ & $\begin{array}{ll}\text { a. A Monthly Income of } \\
\text { 尹132,000 Up Until } \\
\text { 尹220,000+. } \\
\text { b. Spending 尹2,000 - } \\
\text { 尹5,000+ for Internet } \\
\text { Usage Monthly. } \\
\text { c. Knows Little to } \\
\text { Moderate Knowledge on } \\
\text { Bufferbloat, And. } \\
\text { d. Prefers Buying } \\
\text { Technology Products } \\
\text { Physically. }\end{array}$ & $\begin{array}{l}\text { A Flagship Router with the Best } \\
\text { Specifications that are Currently } \\
\text { Available: } \\
\text { Significantly Increased Specifications and } \\
\text { Build Quality: } \\
\text { Single Core, Even Higher Frequency to } \\
\text { Dual Core CPU. } \\
\text { 16-32 MB Flash } \\
\text { 128 MB RAM + } \\
\text { Latest Wi-Fi Standard (802.11ax): } \\
\text { Wireless Protected Access } 3 \text { (WPA 3) } \\
\text { High Powered Antennas: } \\
\text { Maximum of } 30 \text { decibel-milliwatts (dBm) }\end{array}$ \\
\hline
\end{tabular}

\section{Conclusions}

Given the objectives of the research paper at hand, most respondents are unable to find a meaningful answer to bufferbloat and thus provides significant demand for a networking equipment solution at the consumer level. Though reading this paper, one can understand from a sample of respondents in the Philippines that Filipinos are clearly frustrated with their experience when it comes to their internet and there is clearly a prospective market given the significant perceived value for security, latency management, service, customizability, and differentiation.

A guideline has been made for the Philippine context when it comes to the specifications given their income levels and cost of internet use monthly. As mentioned in the literature review, there are substantial quantitative and qualitative benefits when it comes to an optimal implementation 
of Active Queue Management. Should this be implemented on a commercial scale for consumers, this would be of major benefit to consumers which will increase productivity during this pandemic - especially when productivity is already compromised.

Consequently, when the internet infrastructure proves to be robust and extremely responsive, this further allows for the exchange of information on a faster rate. Although the effects of antibufferbloat measures may be not apparent from an individual standpoint immediately, the benefits accumulate over time in the form of saved time, increased productivity, and increased research into the latest standards of networking given the widespread implementation and its clear merits. This improves the sharing of information on a great scale, improving the well-being of a country. By extension, keeping said country competitive in the Fourth Industrial Revolution.

\section{REFERENCES}

[1] Arora, N., \& Singh, G. (2015). Practical Appraisal of Distinguish Active Queue Management Algorithms. International Journal of Computer Science and Mobile Computing, 496-505.

[2] Bufferbloat.net. (n.d.). Cake - Common Applications Kept Enhanced. Retrieved March 8, 2021, from Bufferbloat.net: https://www.bufferbloat.net/projects/codel/wiki/Cake/

[3] C. V. Hollot, V. Misra, D. Towsley and Weibo Gong, "Analysis and design of controllers for AQM routers supporting TCP flows," in IEEE Transactions on Automatic Control, vol. 47, no. 6, pp. 945959, June 2002, doi: 10.1109/TAC.2002.1008360.

[4] Chi, M G. I. (2020). A Study on the Factors Inhibiting High Speed Internet in the Philippines. Retrieved March 8, 2021, from Academia: https://www.academia.edu/44296610/A_Study_on_the_Factors_Inhibiting_High_Speed_Internet_in_ the_Philippines

[5] DSLReports. (n.d.). Bufferbloat. DSLReports. Retrieved April 14, 2021, from http://www.dslreports.com/faq/17883

[6] Kotler, P., \& Armstrong, G. (2018). Principles of Marketing 17th Edition. In P. Kotler, \& G. Armstrong, Principles of Marketing 17th Edition (pp. 603-612). New York: Pearson.

[7] Khatari, M., Zaidan, A., Zaidan, B., Albahri, O., \& Alsalem, M. (2019). Multi-Criteria Evaluation and Benchmarking for Active Queue Management Methods: Open Issues, Challenges and Recommended Pathway Solutions. International Journal of Information Technology and Decision Making, 1187-1242.

[8] Moon, J.; Choe, Y.; Song, H. Determinants of Consumers' Online/Offline Shopping Behaviours during the COVID-19 Pandemic.Int.J. Environ. Res. Public Health 2021,18,1593. https://doi.org/10.3390/ijerph18041593

[9] Mulky, E., Jain, P., Bhatia, S., Dash, S., \& Dutta, R. (2012). Tail Drop Algorithm. Retrieved April 19, 2021, from https://sites.google.com/a/ncsu.edu/tail-drop-vs-red/plan-of-work/tail-drop-algorithm

[10] Nichols, K., Jacobson, V. (6 May 2012). "Controlling Queue Delay". ACM Queue. ACM Publishing. doi:10.1145/2209249.2209264. Retrieved March 8, 2021.

[11] Quan, J., Wang, X., \& Quan, Y. (2019). Effects of Consumers' Strategic Behavior and Psychological Satisfaction on the Retailer's Pricing and Inventory Decisions. IEEE Access, Access, IEEE, 7, 178779-178787. https://doi.org/10.1109/ACCESS.2019.2958685

[12] R. Adams, "Active Queue Management: A Survey," in IEEE Communications Surveys \& Tutorials, vol. 15, no. 3, pp. 1425-1476, Third Quarter 2013, doi: 10.1109/SURV.2012.082212.00018.

[13] R. F. E. Silva and P. M. Carpenter, "Controlling Network Latency in Mixed Hadoop Clusters: Do We Need Active Queue Management?" 2016 IEEE 41st Conference on Local Computer Networks (LCN), Dubai, United Arab Emirates, 2016, pp. 415-423, doi: 10.1109/LCN.2016.70.

[14] Schiffman, L. G., \&Wisenblit, J. (2015). Consumer Behavior: 11th Edition (Eleventh ed.) (pp. 122123, 170-183). Pearson.

[15] Tsourela, M., \&Nerantzaki, D.-M. (2020). An Internet of Things (IoT) Acceptance Model. Assessing Consumer's Behavior toward IoT Products and Applications. Future Internet, 12(11), 1. https://doi.org/10.3390/fi12110191 
[16] White, G., \& Rice, D. (2013, April). Cablelabs Access Network Technologies. Retrieved March 5, 2021, from Cablelabs: https://www-res.cablelabs.com/wpcontent/uploads/2019/02/28094033/Active_Queue_Management_Algorithms_DOCSIS_3_0.pdf

\section{Authors}

Min Guk I. Chi was born in 2001 and graduated High School from Xavier School San Juan in the Philippines. Possessing a love for research, he has successfully completed major research papers for academic requirements independently. Currently, he is taking up 3rd Year Business Administration majoring in entrepreneurship at S P Jain School of Global Management.

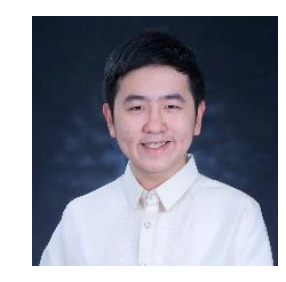

(C) 2021 By AIRCC Publishing Corporation. This article is published under the Creative Commons Attribution (CC BY) license. 